\title{
Neurofeedback: A Noninvasive Treatment for Symptoms of Posttraumatic Stress Disorder in Veterans
}

\author{
Connie J. McReynolds ${ }^{1^{*}}$, Jodi Bell ${ }^{2}$, and Tina M. Lincourt ${ }^{2}$ \\ ${ }^{1}$ California State University, San Bernardino, Institute for Research, Assessment \& Professional Development, San \\ Bernardino, California, USA \\ ${ }^{2}$ Loma Linda University, Department of Psychology, Loma Linda, California, USA
}

\begin{abstract}
This paper discusses positive therapeutic gains made with veterans whose primary treatment for posttraumatic stress disorder (PTSD) was artifact corrected neurofeedback. Assessments completed after both 20 and 40 halfhour sessions of treatment identified significant improvements for both auditory and visual attention using the IVA2 and significant improvements in well-being based on the General Well-Being Scale (GWBS). It was discovered that neurofeedback impacted individuals' overall auditory attention and IVA-2 global auditory test scores significantly improved after both $20(p<.007$, Cohen's $d=0.5)$ and 40 training sessions $(p<.0001$, Cohen's $d=$ $0.8)$. Veterans were found to have significant enhancements in auditory vigilance $(p<.03)$, processing speed $(p$ $<.0009)$ and focus $(p<.01)$. The IVA-2 global measure of visual attention was also found to show significant improvements after 20 sessions $(p<.004$, Cohen's $d=0.5)$ and after 40 sessions $(p<.06$, Cohen's $d=0.4)$. Specific improvements in visual processing speed $(p<.04)$ and focus $(p<.02)$ were identified after 40 sessions. Ratings of well-being significantly improved after treatment $(p<.001$, Cohen's $d=0.8)$ with $84 \%$ of the veterans improving five points or more on the GWBS. Improvements in well-being were found to be significantly correlated with increases in veterans' overall auditory attention $(r=.44, p<.03)$ and auditory processing speed $(r=.57, p$ $<.005)$.
\end{abstract}

Keywords: veterans; posttraumatic stress disorder; PTSD; well-being; IVA-2; CPT; GWBS; attention; artifact corrected; neurofeedback; EEG biofeedback

Citation: McReynolds, C. J., Bell, J., \& Lincourt, T. M. (2017). Neurofeedback: A noninvasive treatment for symptoms of posttraumatic stress disorder in veterans. NeuroRegulation, 4(3-4), 114-124. http://dx.doi.org/10.15540/nr.4.3-4.114

*Address correspondence to: Connie J. McReynolds, PhD, Edited by:

Director, Institute for Research, Assessment \& Professional Rex L. Cannon, PhD, Knoxville Neurofeedback Group, Knoxville, Development, Department of Special Education, Rehabilitation \& Tennessee, USA

Counseling, College of Education, California State University, San Bernardino, 5500 University Parkway, San Bernardino, CA 92407, USA. Email: cmcreyno@csusb.edu

Copyright: $\odot$ 2017. McReynolds et al. This is an Open Access article distributed under the terms of the Creative Commons Attribution License (CC-BY).

Reviewed by:

Rex L. Cannon, PhD, Knoxville Neurofeedback Group, Knoxville, Tennessee, USA

Randall Lyle, PhD, Mount Mercy University, Cedar Rapids, lowa, USA

\section{Introduction}

One in five veterans returning from Iraq and Afghanistan conflicts have been identified as experiencing symptoms of posttraumatic stress disorder (PTSD; RAND, 2008). PTSD indicators can include behavioral, psychological, mood, and sleep symptoms, along with an emotional detachment, or unwanted or intrusive thoughts (NIMH, 2016). Specific symptoms include agitation, irritability, hostility, hypervigilance, self-destructive behavior, social isolation, flashbacks, fear, severe anxiety, or mistrust (American Psychiatric Association, 2013). Moreover, mood indicators can comprise a loss of interest or pleasure in activities, guilt, or loneliness, while sleep dysfunction can involve insomnia or nightmares. Additional symptoms of PTSD include informational processing dysregulation with impairments in attention and working memory (Karl, Malta, \& Maercker, 2006; Mirsky, Anthony, Duncan, 
Ahearn, \& Kellam, 1991; Vasterling et al., 2002), excessive reactivity to trauma-related cues (Buckley, Blanchard, \& Neill, 2000), and physiological responses that trigger the "fight-flight" response (Benson, 1975).

Traditional treatments for PTSD symptoms include pharmacotherapy and/or talk therapy; however, both common approaches have some realistic limitations. Specifically, pharmacotherapy addresses general symptoms but, unfortunately, can have a wide variety of medication side effects and, frequently, does not correct the underlying cause (van der Kolk et al., 2016). Similarly, conventional talk therapy for PTSD, while helpful for some individuals (Breuer \& Freud, 1966), has been found not to be effective with others (Atkinson, 1999; Bisson, Roberts, Andrew, Cooper \& Lewis, 2013; Bradley, Greene, Russ, Dutra, \& Westen, 2005; Demos 2005; NICE, 2005; van der Kolk et al., 2016; Wylie, 2004). The use of traditional talk therapy often focuses on encouraging the person to emotionally recall the traumatic event and even to reexperience it somatically in an effort to reprocess the trauma and relieve its ongoing effects and symptoms. In contrast, the use of neurofeedback treatment avoids the potential triggering of painful experiences pertaining to the traumatic event (Reiter, Andersen, \& Carlsson, 2016; van der Kolk et al. 2016) and instead helps the individual by enhancing their ability to be focused, attentive, and aware in the present moment. Through the use of neurofeedback, the person is able to release the painful experience without reliving the trauma as a means of exorcising it (Robbins, 2000).

It has been advanced that talk therapy may not be very effective for some individuals because the recall of traumatic events can easily initiate the activation of the brain's limbic circuits and provoke a strong emotional reaction that can potentially diminish the functioning of the left frontal lobe for self-regulation (Baum, 1997; Demos, 2005; Thompson \& Thompson, 2003; van der Kolk, McFarlane, \& Weisaeth, 1996; Wylie, 2004). Further, it is believed that for some veterans talk therapy triggers a strong physiological response to past emotional trauma, subsequently diminishing the effectiveness of the talk therapy approach (Benson, 1975; Demos, 2005).

Since it is recognized that memories of a traumatic event can activate the limbic system and be countertherapeutic for a number of veterans (Baum, 1997; van der Kolk et al., 1996; Wylie, 2004), then a viable alternative for the treatment of PTSD is to consider neurofeedback. By using neurofeedback training to decrease activation levels in the limbic system and enhance the self-regulatory capabilities of the frontal lobe system, veterans can experience PTSD symptoms while in a relaxed, focused mental state and use the frontal lobe's ability to process, resolve, and release the traumatic experience (Robbins, 2000; White \& Richards, 2009). A key premise of neurofeedback training is that it is structured to improve cognitive flexibility, physical and mental relaxation, along with greater inner awareness, that can enhance an individual's emotional self-control skills thereby enabling the person to gradually process and release the conditioned reaction to past emotional events (Mason \& Brownback, 2001).

Neurofeedback therapy, or EEG biofeedback, has been widely used for more than 30 years. During this time, it has gained recognition as an acceptable approach for treating conditions ranging from Attention-Deficit/Hyperactivity Disorders (ADHD) to anxiety, depression, sleep disorders, and learning disabilities (Hammond, 2011). Neurofeedback works by helping individuals learn to become more aware and sensitive to their emotional and mental states in order to develop better self-regulation, selfawareness, and attention control, thus allowing for individuals to slowly and safely experience traumatic memories in order to process and decondition their impact without becoming overwhelmed (Demos, 2005; Othmer \& Othmer, 2009). While the initial stage of the neurofeedback therapeutic process for PTSD is to facilitate the development of a calm and stable mental state, the next phase is to permit the brain to access and to resolve the emotional expression of underlying traumas through deconditioning of emotional reactions that previously occurred whenever they spontaneously arose or were triggered by environmental stimuli (Robbins, 2000).

Neurofeedback has been found in research studies to be clinically effective and comparable in outcome measures to other recognized types of treatments for individuals who experience the symptoms of PTSD (Peniston \& Kulkosky, 1991; van der Kolk et al., 2016). Weaknesses involving sustained attention have been identified previously in individuals with PTSD (Sachinvala et al., 2000), and documented specifically in veterans with PTSD (Vasterling, Brailey, Constans \& Sutker, 1998; Vasterling et al., 2002; Uddo, Vasterling, Brailey, \& Sutker, 1993), making the use of neurofeedback particularly beneficial in treating the attentional dysfunction, which is often prevalent in PTSD 
populations. Neurofeedback can be conceptualized as utilizing the brain's inherent capability of neuroplasticity that allows individuals to become aware of the faint cues of their EEG neural activity. By attending to the feedback provided, individuals learn to control and direct their brain activity in order to cultivate a more harmonious and balanced mental state (Budzynski, 1999; Demos, 2005; Nunez, 1981; Othmer \& Othmer, 2009; Speckmann \& Elger, 1987; White \& Richards, 2009). The changes resulting from neurofeedback have been found to result in long-term changes and positive outcomes (Budzynski, 1999; Demos, 2005; Othmer \& Othmer, 2009).

The training process involves placing EEG sensors over selected brain regions on the scalp and ears to measure the amplitude of the electrical activity of the brain's neuronal network. The individual's brainwave patterns are quantified and then displayed on a computer screen in a meaningful manner using both visual and auditory feedback. The therapist develops a treatment plan, which can consist of 20 to 40 training sessions lasting about thirty minutes each, and establishes therapeutic goals that are specific for each person's needs. Both visual and auditory game-like feedback is utilized to reinforce the achievement of training goals.

The purpose of this retroactive study was to evaluate the clinical effectiveness of the neurofeedback treatment for the 20 veterans who presented with a variety of PTSD symptoms including anxiety, panic attacks, concentration difficulties, sleep disorders, depression, and memory concerns. It was hypothesized that the Integrated Visual and Auditory Continuous Performance Test Version 2 (IVA-2 CPT) global measures of visual and auditory attention (VAQ and AAQ scale scores, respectively) would show a significant improvement after both 20 and 40 sessions of treatment. A second hypothesis was that the ratings of well-being measured by the General Well-Being Scale (GWBS) would significantly increase after 40 sessions were completed. Since five statistical tests were planned and neurofeedback training was expected based on past studies to positively affect attention and wellbeing, a one-tail alpha level was set to $p<.02$ based on the Bonferroni correction with adjustments for the initial mean correlation between all test scales. Additional analyses were planned to examine the relationship between improvements in IVA-2 measures of attention and the GWBS ratings of wellbeing in order to explore in detail the specific aspects of attentional functioning that changed after veterans completed 20 and 40 neurofeedback sessions and whether or not improvements in attention led to increases in veterans' feelings of well-being.

\section{Methods}

\section{Participants}

Neurofeedback treatment was provided for 20 U.S. military veterans (16 males, 4 females). The average age of the veterans at the time of testing was 46 years old $( \pm 1 S D=17.7)$. The self-reported primary diagnoses of these veterans included PTSD (65\%), ADHD (15\%), Major Depression (10\%), Generalized Anxiety (5\%), and Learning Disability $(5 \%)$. The participants for this study were randomly drawn from an archival database of a sample of veterans who had previously received neurofeedback training. Individualized neurofeedback training was provided within a university-based clinic setting. Veterans were not compensated to participate in the neurofeedback training. The funding agency provided support for neurofeedback services to be delivered as a clinical intervention rather than as a study of a specific neurofeedback protocol. This study was approved by the California State University San Bernardino Internal Review Board. Participants were provided with an informed consent process.

\section{Measurements}

The IVA-2 CPT has been found to be a valid and reliable measure of both visual and auditory attention functioning in both children and adults and provides both global and primary measures of attentional functioning. The normative sample, with approximately equal numbers of males and females, included 1,700 individuals ages 6 to 96 (Maddux, 2010). The scales on the IVA-2 have a mean of 100 and a standard deviation of 15 . The IVA-2 global measures of attention used in this study are the Visual Attention Quotient (VAQ) and the Auditory Attention Quotient (AAQ). The $V A Q$ is a global measure of attention that is comprised of three primary visual scales: Vigilance, Speed, and Focus. Vigilance measures errors of omission, and Speed provides a measure response time to visual test targets. Focus is a measure of the variability of response time to visual test targets. The AAQ has the exact same components and differs in that it assesses auditory test responses to the same primary measures of attention (Sandford \& Sandford 2015). Moreover, the IVA-2 has been demonstrated to be valid for adults with neurological insults such as traumatic brain injury (TBI; Tinius, 2003). 
The GWBS is an 18-item questionnaire that is a selfreport rating scale that measures a person's general sense of well-being. It incorporates six subscales of well-being including measures of anxiety, positive well-being, depression, vitality, general health, and self-control. The GWBS has been found to be both a valid and reliable measure of well-being for several ethnic minority groups including young Caucasian males (Fazio, 1977) along with Japanese (Nakayama, Toyoda, Ohno, Yoshiike, \& Futagami, 2000), Mexican-American (Poston et al., 1998), and African-American populations (Taylor et al., 2003).

\section{Test Procedure}

Every veteran was administered and completed the IVA-2 CPT and the GWBS before beginning their first neurofeedback session. Testing was individually administered and scored in accordance with test procedures. There were a few individuals who were not able to validly respond to either visual or auditory IVA-2 test stimuli due to their extreme deficits in attentional functioning. In these cases, their "invalid scores" for IVA-2 were scored as zero in accordance with the test interpretive procedures (Sandford \& Sandford, 2015). After the completion of 20 and again after 40 neurofeedback sessions, the IVA-2 test was readministered. Twenty veterans completed 20 neurofeedback sessions and 19 completed an additional 20 sessions. One individual dropped out due to scheduling conflicts after completing 20 sessions. Following the last neurofeedback session, the GWBS rating scale was administered for the second time. IVA-2 data was analyzed comparing baseline test scores and the scores obtained after both 20 and 40 sessions were completed. The GWBS rating scale score analysis compared pretraining baseline scores to scores obtained after 40 sessions of treatment.

\section{Neurofeedback Treatment Protocols}

An individualized neurofeedback training plan was developed for each participant and clinically modified as necessary. Therapeutic goals focused on improving attentional functioning and reducing any identified mental stress related to the symptoms of depression and anxiety. Training was completed using the SmartMind 3 artifact corrected neurofeedback system with a two-channel EEG station (BrainTrain, Inc., North Chesterfield, VA) which continuously filters out frequently occurring, very brief EMG artifacts in real time without interrupting the training program. Neurofeedback exercises were provided in game-like format that utilized both visual and auditory reinforcement, as well as graphs and numerical scores to provide positive reinforcement. The first step in the training session was to collect an individual's baseline EEG data in order to determine Z-Score feedback goals for each individual. Based on each individual's performance, they were provided clinically relevant feedback and adjustments were made to the training protocol to optimize their performance. All EEG data was automatically recorded.

\section{Results}

Since five main tests were required to answer the hypotheses of this study, the alpha level was determined to be .02 using a Bonferroni correction adjusted for the pretreatment correlation of the measures used $(r=.46)$. All $t$-tests were one-tail measures given that it was expected based on past research studies that neurofeedback would result in positive changes in attention and emotional selfregulation. Given that the normative mean quotient score of the IVA-2 test is 100 and its standard deviation is 15 , any increase of eight or more quotient score points (i.e., greater than one half of a standard deviation) is considered clinically significant. This section will first address the main hypotheses. Next, more specific IVA-2 component measures of auditory and visual attention will be examined in an exploratory analysis with the alpha level set to .10 in order to explore more in-depth any changes in attention and their relationship to improvements in well-being.

In order to evaluate whether or not neurofeedback training improves auditory and visual attention, paired sample $t$-tests were computed comparing pretreatment IVA-2 AAQ and VAQ quotient test scores with each individual's IVA-2 test scores after completing 20 and then 40 sessions. As indicated in Table 1 , veterans $(N=20)$ significantly increased their AAQ score after 20 sessions of treatment from a mean of 83 (Mildly Impaired) to 96 (Average), a 13-point increase, $t(19)=-2.68, p<.007$, Cohen's $d$ $=0.5$. AAQ scores were also found to be significantly higher after 40 treatment sessions (see Table 2, $N=19$ ) and increased from 82 to 100 , an 18-point improvement, $t(18)=-4.53, p<.0001$, Cohen's $d=0.8$. 
Table 1

Paired Sample t-tests comparing mean IVA-2 Quotient scale scores at Baseline and after veterans $(N=20)$ completed 20 neurofeedback training sessions.

\begin{tabular}{ccccccc}
\hline IVA-2 Attention Scales & $\begin{array}{c}\text { Baseline } \\
(N=20)\end{array}$ & $\begin{array}{c}20 \\
\text { Sessions }\end{array}$ & $\begin{array}{c}\text { Q Score } \\
\text { Change }\end{array}$ & $\begin{array}{c}\text { Pooled } \\
\text { SD }\end{array}$ & Sig. & $\begin{array}{c}\text { Cohen's } \\
d\end{array}$ \\
\hline Auditory Attention Quotient & 83 & 96 & 13 & 24 & 0.007 & 0.5 \\
Auditory Vigilance & 88 & 97 & 9 & 28 & 0.15 & n.a. \\
Auditory Speed & 84 & 100 & 16 & 22 & 0.002 & 0.7 \\
Auditory Focus & 91 & 94 & 3 & 17 & 0.21 & n.a. \\
Visual Attention Quotient & 84 & 96 & 12 & 27 & 0.004 & 0.5 \\
Visual Vigilance & 86 & 94 & 8 & 28 & 0.08 & 0.3 \\
Visual Speed & 92 & 103 & 13 & 22 & 0.03 & 0.5 \\
Visual Focus & 84 & 96 & 12 & 26 & 0.007 & 0.5 \\
\hline
\end{tabular}

Table 2

Paired Sample t-tests comparing mean IVA-2 Quotient scale scores at Baseline and after veterans ( $N=19)$ completed 40 neurofeedback training sessions.

\begin{tabular}{ccccccc}
\hline IVA-2 Attention Scales & $\begin{array}{c}\text { Baseline } \\
(N=19)\end{array}$ & $\begin{array}{c}40 \\
\text { Sessions }\end{array}$ & $\begin{array}{c}\text { Q Score } \\
\text { Change }\end{array}$ & $\begin{array}{c}\text { Pooled } \\
\text { SD }\end{array}$ & Sig. & $\begin{array}{c}\text { Cohen's } \\
d\end{array}$ \\
\hline Auditory Attention Quotient & 82 & 100 & 18 & 24 & 0.0001 & 0.8 \\
Auditory Vigilance & 87 & 100 & 13 & 27 & 0.03 & 0.5 \\
Auditory Speed & 82 & 102 & 20 & 23 & 0.0009 & 0.9 \\
Auditory Focus & 91 & 97 & 6 & 14 & 0.01 & 0.5 \\
Visual Attention Quotient & 84 & 95 & 11 & 31 & 0.06 & 0.4 \\
Visual Vigilance & 87 & 90 & 3 & 35 & 0.37 & n.a. \\
Visual Speed & 90 & 102 & 12 & 22 & 0.04 & 0.5 \\
Visual Focus & 83 & 97 & 14 & 25 & 0.02 & 0.6 \\
\hline
\end{tabular}

In Figure 1, the continued improvement in auditory attention from 20 to 40 sessions that was significant can be viewed, $t(18)=-1.83, p<.04$, Cohen's $d=$ 0.2. The IVA-2 VAQ test scores significantly increased 12 points after 20 sessions, $t(19)=-2.99$, $p<.004$, Cohen's $d=0.5$; and 11 points after 40 sessions, $t(18)=-1.64, p<.06$, Cohen's $d=0.4$. Unlike AAQ scores, VAQ measures were not found to significantly change after an additional 20 training sessions as seen in Figure 1. Thus, these test results support the hypothesis that neurofeedback training led to a significant improvement in global measures of both auditory and visual attention.

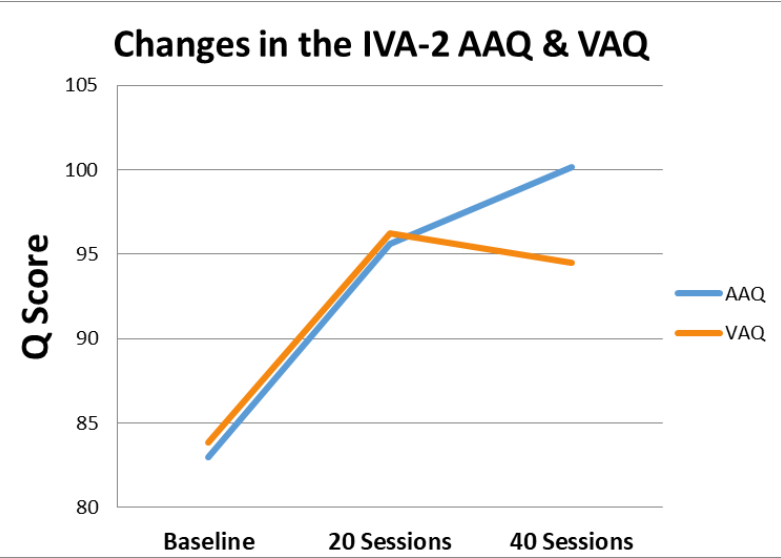

Figure 1. Changes in the IVA-2 Auditory Attention (AAQ) and Visual Attention (VAQ) standard $Q$ scale scores after 20 and 40 sessions of neurofeedback. 
An examination of the changes in $A A Q$ and $V A Q$ on a clinical basis was completed to further explore and predict the potential benefit of neurofeedback on an individual basis. In order to do so, a positive or negative change in IVA-2 quotient scale scores of eight or more was considered clinically significant. After 20 sessions, $80 \%$ of the veterans improved in either AAQ or VAQ scores by eight points or more and for 40 sessions the treatment success rate was $74 \%$. IVA-2 testing after 20 sessions found that $15 \%$ did not improve or declined (greater than eight points) in either AAQ or VAQ scores, and at 40 sessions, $10 \%$ still did not show any change in their attentional functioning. Only one person, or $5 \%$ of the veterans at 20 sessions, decreased significantly in his VAQ score and had no meaningful change in $A A Q$ indicating that he was more impaired in visual attention when evaluated for the second time. At 40 sessions, three individuals performed significantly more poorly in respect to visual attention and had no improvement or decrement in their auditory attention. In general terms, these results indicate that it is reasonable to expect that about four out of five veterans will significantly benefit from neurofeedback training, but that about 1 out of 10 will actually decline (eight or more points) in their visual attention without any compensating improvement in their auditory attention.

Changes in self-reports of well-being were assessed by comparing the initial scores on the GWBS with rating scores obtained after 40 sessions of treatment were completed using a paired sample $t$-test.

\section{Table 3}

Paired Sample t-Test comparing the GWBS Well-Being rating scale scores at Baseline and after veterans $(N=19)$ completing 40 neurofeedback training sessions.

\begin{tabular}{lcccccc}
\hline $\begin{array}{l}\text { GWBS Well-Being } \\
\text { Rating Scale }\end{array}$ & $\begin{array}{c}\text { Baseline } \\
(N=19)\end{array}$ & $\begin{array}{c}40 \\
\text { Sessions }\end{array}$ & $\begin{array}{c}\text { Q Score } \\
\text { Change }\end{array}$ & $\begin{array}{c}\text { Pooled } \\
\text { SD }\end{array}$ & Sig. & $\begin{array}{c}\text { Cohen's } \\
d\end{array}$ \\
\hline GWBS Rating Scale Score & 58 & 72 & 14 & 24 & 0.001 & 0.8 \\
\hline
\end{tabular}

As can be seen in Table 3, the GWBS rating scale scores significantly improved 14 points from 58 to $72, t(18)=-3.55, p<.001$, Cohen's $d=0.8$. A positive change of five points or more in the GWBS total score was found for $84 \%$ of the participants. The changes are graphed in Figure 2.

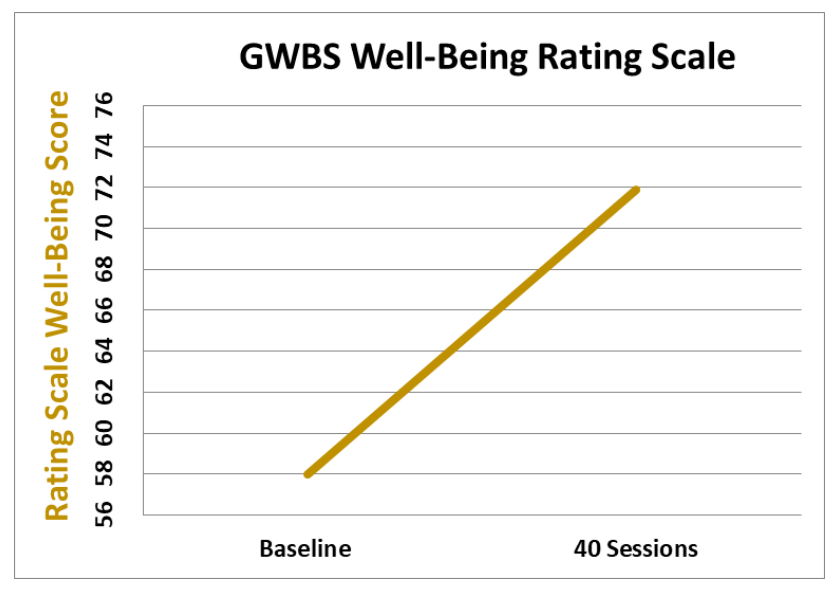

Figure 2. Changes in the General Well-Being Scale (GWBS) after veterans completed 40 neurofeedback sessions.
The GWBS has three primary interpretive categories for labeling a person's score: Severe Distress (0$60)$, Moderate Distress (61-72) and Positive Wellbeing (73-110). Initially, $79 \%$ of the individuals in this study, who completed it, rated themselves as either experiencing severe or moderate distress and $21 \%$ reported having scores reflective of positive well-being which they maintained during this study. Distress was defined as an "inner personal state" with elevated feelings of anxiety and depression combined with limited reports of good general health, vitality, positive well-being, and the ability for self-control (Dupuy, 1977). To evaluate the clinical effects of treatment changes, significant improvements in well-being were defined as a change from a more impaired level of distress to less impaired using the category labels provided above from the test manual. Of the 15 individuals who were identified as having either severe or moderate levels of distress prior to treatment, nine (60\%) significantly improved in their well-being and seven of these nine veterans $(78 \%)$ rated themselves as having a positive state of well-being after completing treatment. One veteran became clinically worse $(7 \%)$ and the five individuals (33\%) did not change in their ratings of well-being. These results show that neurofeedback is likely to help 6 out of 10 veterans 
improve their general well-being and that about 5 out of these 6 individuals who have severe to moderate levels of distress prior to treatment are likely to return to a healthy state of positive well-being after neurofeedback.

In Table 4, the correlations between the GWBS rating scores and the IVA-2 global and primary measures of auditory and visual attention completed after treatment are reported. The question of interest was whether or not improvements in either auditory or visual attention contributed to increases in an individual's feelings of well-being. Prior to any treatment, the correlations between the first GWBS rating scale scores and the IVA-2 CPT test scores were examined and no significant correlations were found. After neurofeedback treatment, a significant correlation of .44 $(p<.03)$ was found for the global AAQ, which consists of the Vigilance, Speed and Focus primary scale scores. The auditory Speed scale, which is a measure of the discriminatory response time to the IVA-2 targets (i.e., click if you hear the number one), was found to have a significant correlation of .57 $(p<.005)$ with the GWBS. No significant correlations were identified for any IVA-2 visual scale.

\section{Table 4}

Correlations of the IVA-2 Attention Scales and the GWBS After Veterans Completed 40 Sessions of Neurofeedback Training.

\begin{tabular}{lcc}
\hline IVA Attention Scales & $\begin{array}{c}\text { GWBS Rating } \\
\text { Scores }\end{array}$ & Sig. \\
\hline Auditory Attention Quotient (AAQ) & $\mathbf{0 . 4 4}$ & 0.03 \\
Auditory Vigilance & 0.28 & n.s. \\
Auditory Speed & $\mathbf{0 . 5 7}$ & 0.005 \\
Auditory Focus & 0.28 & n.s. \\
Visual Attention Quotient (VAQ) & 0.17 & n.s. \\
Visual Vigilance & 0.14 & n.s. \\
Visual Speed & 0.16 & n.s. \\
Visual Focus & 0.00 & n.s. \\
\hline
\end{tabular}

Tables 1 and 2 are useful in that they show that prior to treatment (i.e., baseline) the mean attention scale scores for both auditory and visual were in what is labeled as a mild impairment. After neurofeedback treatment was completed, all global and primary IVA-2 scale scores fell for the most part in the middle of the Average range. After 20 sessions, the four measures of visual attention appeared to reach a maximum level of improvement and continued training did not seem to lead to any further changes in visual attention with mean scale scores remaining well within the average range and effect sizes essentially being equivalent. In contrast, continued neurofeedback training did seem to strengthen the attention skills of participants. To support this conclusion, it can be seen that the total $Q$ score change after 20 more training sessions for the four auditory scales increased by 16 points (39\%). In addition, the effect size after 20 sessions was medium for two scales and nil for the other two scales, because those two scales did not significantly improve. But after 40 sessions, all four auditory scales were found to significantly improve and the effect sizes were identified to be large for both $A A Q$ and Speed scales and medium for Vigilance and Focus. It is interesting to note that the only two significant correlations between the IVA-2 scales and the GWBS discussed above were the $\mathrm{AAQ}$ and Speed scales, which after 40 sessions showed large effect sizes.

Given this study was archival, EEG protocols were individualized and modified as determined appropriate by the clinicians working with the veterans. Consequently, any statistical analysis on a group basis in order to examine possible EEG learning effects was not possible due to the fact that the clinically selected training protocols varied and were modified by clinicians during the course of the treatment in order to maximize the learning process for each individual. The agency providing support for these neurofeedback services did so with the understanding that services were provided on an individualized basis and not as a research study to evaluate a specific fixed neurofeedback training protocol.

\section{Discussion}

The positive benefits of neurofeedback as a therapeutic intervention for helping reduce PTSD symptomatology have been reported in a number of studies discussed above (Othmer \& Othmer, 2009; Peniston \& Kulkosky, 1991; van der Kolk et al. 2016). This study specifically identified that artifact corrected neurofeedback, which works by filtering out the contamination that continually results from naturally occurring EMG artifacts such as eye blinks, eye movements and facial activity, significantly improved both auditory and visual attention as measured by the IVA-2. As a group, these individuals initially presented with mild attentional impairments. After 20 half-hour treatment sessions, both their auditory and visual attention abilities were 
normalized with standardized scale scores falling in the middle of the average range and effect sizes in the medium range.

While this study utilized archival data and there was no control group to control for possible test practice effects, the IVA-2 is an objective measure of attention which controls for practice effects in both its simplistic design (i.e., the test rule is to click if you see or hear the number one) and in its pretest instruction phase, which includes specific opportunities for individuals to practice the test before taking it. The reliability study in the test manual found that on retesting subjects did not significantly change by more than three to four points in either direction (Sandford \& Sandford, 2015). Thus, any group increases in IVA-2 quotient scores greater than three to four points can be validly interpreted as a result of an active treatment and not due to practice effects. In this study, it was found that 20 additional neurofeedback sessions led specifically to the significant enhancement of auditory attention as evidenced by the greater effect sizes observed and the significant increase in the AAQ from 20 to 40 sessions (see Tables 1 and 2). While visual attention significantly improved after 20 sessions, no further improvements in visual scores were observed after training continued for an additional 20 sessions. Thus, the first hypothesis of this study that neurofeedback would significantly improve both auditory and visual attention was confirmed and effect sizes were large for the enhancement of auditory attention and medium in respect to visual attention. Given that the effects of neurofeedback for individuals with PTSD resulted in a greater enhancement of auditory than visual attention leads to the recommendation that the assessment of the effects of neurofeedback will need to include both auditory and visual measures of attention.

Given the nature of their missions, U.S. military veterans are subjected to exceptionally traumatic events. These traumatic events can result in an ongoing inner state of severe to moderate distress stemming from the numerous symptoms of PTSD. Consequently, any treatment to help reduce the emotional dysregulation and promote a greater sense of well-being will need to use an assessment tool like the GWBS to evaluate treatment effects. In this study, significant improvements in well-being were achieved which showed a large effect size after 40 sessions of neurofeedback were completed. While the lack of a control group in this study limits the conclusion that neurofeedback was the primary causal factor for the observed improvement in well- being, the discovery that the global measure of $A A Q$ and specifically discriminatory auditory response time (i.e., the auditory Speed scale) were significantly correlated with ratings of well-being after 40 sessions of neurofeedback, but not prior to training, lends support to the validity of neurofeedback being the key factor in the improvements observed in well-being. This conclusion is further strengthened by the fact that the greatest size effects were found for the AAQ and auditory Speed scales after treatment was completed. This suggests that neurofeedback led to improvements in well-being in a specific way. Increases in auditory attention may help improve listening skills and an individual's ability to engage more effectively in verbal and, thus, social communication. When an individual can be a better listener and can understand what others are verbally communicating, then both positive social interaction and social feedback from others is more likely to occur leading to greater inner feelings of well-being. This reasoning would need to be further explored in additional studies by measuring improvements in social interaction, listening skills, and the ability to better process auditory information. The second hypothesis that neurofeedback would lead to significant increases in well-being for individuals with PTSD was supported by these results, along with indications that the mechanism may at least in part relate to improvements specifically in auditory attention and auditory processing speed.

Identifying multimodal approaches to treat PTSD creates opportunities for optimal patient care. Further research needs to explore the potential for neurofeedback to be used in combination with cognitive behavioral therapy and other interventions that have been found to improve the emotional and behavioral functioning and coping skills for individuals experiencing symptoms of PTSD. If the person's attentional functioning can be restored to premorbid levels along with an increase in feelings of well-being, then other therapeutic interventions may synergistically combine to maximize clinically targeted goals in less time. In general, given that clients often have varied responses and outcomes to different treatment approaches, a comprehensive and multifaceted treatment approach is called for in order to develop new and more clinically efficacious treatment strategies for PTSD. Neurofeedback offers the potential as an alternative treatment approach that is gradually becoming more widely accepted by many mental health care professionals and warrants institutional and governmental support for new research specifically with veterans who have PTSD based on the results of this study and 
numerous others. Providing neurofeedback to veterans with PTSD who have not been successfully treated with other approaches including medication, provides a means to improve attention, can help reduce abnormal symptomology, improve the person's well-being, increase their ability to tolerate stress, and enable them to develop new skill sets in combination with other evidence-based treatment methods.

This research finding further substantiates the value and benefit of utilizing this new artifact corrected type of neurofeedback in the treatment of veterans with PTSD and warrants further research as a neurofeedback intervention. This study found that neurofeedback helped 4 out of 5 participants clinically improve their auditory and visual attention in only 20 sessions. In this study, initially 8 out of 10 veterans were found to be experiencing severe to moderate levels of distress. After receiving neurofeedback treatment without any supportive counseling or coaching, $60 \%$ of them reported significant clinical improvements in well-being. Thus, the benefits of neurofeedback showed its potential to help veterans improve their psychosocial functioning in ways that generalize to benefit them in everyday life situations.

In interpreting the results of this study, certain limitations were considered. Unquestionably, its archival nature was a primary drawback. Unfortunately, a comprehensive diagnostic intake was not possible due to funding limitations. It would be best to utilize the criterion specified in the DSM- 5 (Diagnostic and Statistical Manual of Mental Disorders, Fifth Edition) in order to accurately diagnose PTSD and differentiate it from other mental disorders. It would also be useful to clarify a veteran's relevant history pertaining to their trauma experiences given that a large number of the individuals participating in this study pilot had been exposed to battlefield trauma at various times, but the length of time between trauma exposure and its severity was unknown. Future research exploring the benefits of neurofeedback in the treatment of the effects of PTSD would benefit from the use of standardized clinical interviews as part of the intake process. In addition, the systematic garnering of symptomatology in more detail is warranted along with comprehensive pre- and postneuropsychological testing in order to evaluate the benefits of neurofeedback in more depth. Six-month and one-year follow-up evaluations to determine the long-term effects of neurofeedback treatment are also components recommended for consideration for future studies in this field. The inclusion of the above additions to future research will help elucidate both the benefits and underlying mechanisms whereby neurofeedback protocols can be evaluated and enhanced to improve their clinical impact. As such, prospective studies will provide a more reliable method of assessing the efficacy of neurofeedback on PTSD symptomology.

While this archival research was not designed to evaluate learning effects, the evaluation and demonstration of individuals' learned control of brainwave activity is an important issue which needs to be addressed in future research. Thus, research specifically designed to measure learning effects respective to the targeted EEG frequencies trained is recommended. However, in order to evaluate learning effects this type of study would require that all participants in the study receive training that used the same standardized treatment protocol for each person. Any clinical modifications to meet a person's specific needs would not be permitted. Consequently, participants would need to be clearly informed that the requirements of the research design are such that the neurofeedback training protocol will not be modified during training and, hence, will not be clinically adjusted to meet an individual's specific needs.

\section{Conclusion}

U.S. military veterans have historically experienced combat-related PTSD. Neurofeedback is becoming more widely used to treat a variety of psychiatric disorders and as such has been used by our clinic to treat veterans experiencing symptoms of PTSD. The results of this study supported the hypothesis that neurofeedback would significantly improve both auditory and visual attention of veterans with PTSD symptomatology. The veterans' improvement in their auditory and visual attention scores revealed that 8 out of 10 of them achieved clinically relevant improvements after only 20 half-hour treatment sessions. The treatment effect sizes of medium to large for this artifact corrected neurofeedback also served to support the clinical efficacy of this type of neurofeedback in improving attention.

A second hypothesis that neurofeedback treatment would significantly improve ratings of well-being was also supported by significant increases in the GWBS rating scales, as well as the large size effect found for this improvement. Only after treatment was completed did the enhancement observed in auditory attention and auditory processing speed correlate with the GWBS rating scores of well-being. The large effect size of the improvement in well- 
being was believed to be attributable to the significant improvements in auditory attention and speed. These two aspects of attention were conceptualized as improving listening and verbal communication skills and, thus, suggested an underlying mechanism for how neurofeedback improves positive well-being. Overall, $60 \%$ of veterans who were initially experiencing severe to moderate levels of distress were identified to have clinically improved as a result of neurofeedback treatment in their well-being and the majority of them $(78 \%)$ were reporting positive levels of well-being after completing treatment. The overall findings of this study support that artifact corrected neurofeedback is a clinically efficacious intervention that helps normalize the mild attentional impairments symptomatic of PTSD and that these specific improvements in auditory attention and processing speed are likely to contribute to better verbal communication skills and enhancing more positive feelings of well-being.

\section{Author Note}

Funding for this intervention was provided by San Bernardino County Veterans Affairs, California agency; all veterans volunteered for this treatment and were not compensated.

\section{References}

American Psychiatric Association. (2013). Diagnostic and statistical manual of mental disorders (5th ed.). Washington, DC: Author. www.psychiatry.org/psychiatrists/practice/dsm

Atkinson, B. J. (1999). The emotional imperative: Psychotherapists cannot afford to ignore the primacy of the limbic brain. Family Therapy Networker, 23(4), 22-33. http://www.thecouplesclinic.com/pdf/emotional_imperative.pdf

Baum, B. (1997). The healing dimensions: Revolving trauma in the body, mind and spirit (pp. 1-114). Tucson, AZ: Healing Dimensions.

Benson, H. (1975). The relaxation response (pp. 16-18, 66-74). New York, NY: William Morrow.

Bisson, J., Roberts, N. P., Andrew, M., Cooper, R., \& Lewis, C. (2013). Psychological therapies for chronic post-traumatic stress disorder (PTSD) in adults. Cochrane Database of System Reviews, $12 . \quad$ http://dx.doi.org/10.1002 /14651858.CD003388.pub4

Bradley, R., Greene, J., Russ, E., Dutra, L., \& Westen, D. (2005). A multidimensional meta-analysis of psychotherapy for PTSD. American Journal of Psychiatry, 162(2), 214-227. http://dx.doi.org/10.1176/appi.ajp.162.2.214

Breuer, J., \& Freud, S. (1966). Studies on hysteria (pp. 55-82). New York, NY: Basic Books.

Buckley, T. C., Blanchard, E. B., \& Neill, W. T. (2000). Information processing and PTSD: A review of the empirical literature. Clinical Psychology Review, 20(8), 1041-1065. http://dx.doi.org/10.1016/S0272-7358(99)00030-6

Budzynski, T. H. (1999). From EEG to neurofeedback. In J. R. Evans \& A. Abarbanel (Eds.), Introduction to Quantitative EEG and Neurofeedback (pp. 65-79). San Diego, CA: Academic Press.

Demos, J. N. (2005). Getting started with neurofeedback. New York, NY: W. W. Norton \& Company.
Dupuy, H. J. (1977). The General Well-being Schedule. In I. McDowell \& C. Newell (Eds.), Measuring health: A guide to rating scales and questionnaires (2nd ed., pp. 206-213). New York, NY: Oxford University Press.

Fazio, A. F. (1977). A concurrent validational study of the NCHS General Well-Being Schedule (DHEW Publication No. [HRA] 78-1347). Hyattsville, MD: U.S. Department of Health, Education, and Welfare, National Center for Health Statistics, Vital \& Health Statistics, Series 2, No. 73. https://www.cdc.gov/nchs/data/series/sr_02/sr02_073.pdf

Hammond, D. C. (2011). What is neurofeedback: An update. Journal of Neurotherapy, 15(4), 305-336. http://dx.doi.org /10.1080/10874208.2011.623090

Karl, A., Malta, L. S., \& Maercker, A. (2006). Meta-analytic review of event-related potential studies in post-traumatic stress disorder. Biological Psychology, 71(2), 123-147. http://dx.doi.org/10.1016/j.biopsycho.2005.03.004

Maddux, C. D. (2010). [Review of the IVA+Plus: Integrated Visual and Auditory Continuous Performance Test]. In R. A. Spies, J. F. Carlson, \& K. F. Geisinger (Eds.), The eighteenth mental measurements yearbook (pp. 434-437). Lincoln, NE: Buros Institute of Mental Measurements.

Mason, L. A., \& Brownback, T. S. (2001). Optimal functioning training with EEG biofeedback for clinical populations: A case study. Journal of Neurotherapy, 5(1-2), 33-42. http://dx.doi.org/10.1300/J184v05n01_04

Mirsky, A. F., Anthony, B. J., Duncan, C. C., Ahearn, M. B., \& Kellam, S. G. (1991). Analysis of the elements of attention: A neuropsychological approach. Neuropsychology Review, 2(2), 109-145. http://dx.doi.org/10.1007/BF01109051

Nakayama, T., Toyoda, H., Ohno, K., Yoshiike, N., \& Futagami, T. (2000). Validity, reliability and acceptability of the Japanese version of the General Well-Being Schedule (GWBS). Quality of Life Research, 9(5), 529-539. http://dx.doi.org/10.1023 IA:1008940902849

National Institute of Clinical Excellence (NICE). (2005). Posttraumatic stress disorder: Management. Retrieved from http://nice.org.uk/guidance/cg26

NIMH. (2016, February). Post-Traumatic Stress Disorder. Retrieved from https://www.nimh.nih.gov/health/topics/posttraumatic-stress-disorder-ptsd/index.shtml

Nunez, P. L. (1981). Electric fields of the brain (pp. 349-400). New York, NY: Oxford University Press.

Othmer, S., \& Othmer, S. F. (2009). Posttraumatic stress disorder - The neurofeedback remedy. Biofeedback, 37(1), 24-31. http://dx.doi.org/10.5298/1081-5937-37.1.24

Peniston, E. G., \& Kulkosky, P. J. (1991). Alpha-theta brainwave neurofeedback therapy for Vietnam veterans with combatrelated post-traumatic stress disorder. Medical Psychotherapy, 4, 47-60. http://michiganbraincoretherapy.com/storage/PTSD /PENISTONPTSD.pdf

Poston, W. S. C., II, Olvera, N. E., Yanez, C., Haddock, C. K., Dunn, J. K., Hanis, C. L., \& Foreyt, J. P. (1998). Evaluation of the factor structure and psychometric characteristics of the General Well-Being Schedule (GWB) with Mexican American women. Women \& Health, 27(3), 51-64. http://dx.doi.org /10.1300/J013v27n03 04

RAND. (2008, April 17.) One in Five Iraq and Afghanistan Veterans Suffer from PTSD or Major Depression. Retrieved from http://www.rand.org/news/press/2008/04/17.html

Reiter, K., Andersen, S. B., \& Carlsson, J. (2016). Neurofeedback treatment and posttraumatic stress disorder: Effectiveness of neurofeedback on posttraumatic stress disorder and the optimal choice of protocol. Journal of Nervous and Mental Disease, 204(2), 69-77. http://dx.doi.org/10.1097/NMD.0000000000000418

Robbins, J. (2000). A symphony in the brain (pp. 160-162). New York, NY: Atlantic Monthly Press. 
Sachinvala, N., von Scotti, H., McGuire, M., Fairbanks, L., Bakst, K., McGuire, M., \& Brown, N. (2000). Memory, attention, function, and mood among patients with chronic posttraumatic stress disorder. Journal of Nervous and Mental Disease, 188(12), 818-823. http://dx.doi.org/10.1097 /00005053-200012000-00005

Sandford, J. A., \& Sandford, S. E. (2015). IVA-2 Integrated Visual and Auditory Continuous Performance Test Manual. North Chesterfield, VA: BrainTrain, Inc.

SmartMind 3 [Computer software and hardware]. (2015). North Chesterfield, VA: BrainTrain, Inc.

Speckmann, E.-J., \& Elger, C. E. (1987). Neurophysiologic basis of the EEG and DC potentials. In E. Niedermeyer \& F. H. Lopes da Silva, F. (Eds.) Electroencephalography (2nd ed., Ch. 2, pp. 1-16). Baltimore, MD: Urban \& Schwarzenberg.

Taylor, J. E., Poston W. S. C., II, Haddock, C. K., Blackburn, G. L., Heber, D., Heymsfield, S. B., \& Foreyt, J. P. (2003). Psychometric characteristics of the General Well-Being Schedule (GWB) with African-American women. Quality of Life Research, 12(1), 31-39. https://link.springer.com/article /10.1023/A:1022052804109

Thompson, M., \& Thompson, L. (2003). The neurofeedback book: An introduction to basic concepts in applied psychophysiology (pp. 70-108). White Ridge, CO: The Association for Applied Psychophysiology and Biofeedback.

Tinius, T. P. (2003). The Integrated Visual and Auditory Continuous Performance Test as a neuropsychological measure. Archives of Clinical Neuropsychology, 18(5), 439454. http://dx.doi.org/10.1016/S0887-6177(02)00144-0

Uddo, M., Vasterling, J. J., Brailey, K., \& Sutker, P. B. (1993). Memory and attention in combat-related post-traumatic stress disorder (PTSD). Journal of Psychopathology and Behavioral Assessment, 15(1), 43-52. http://dx.doi.org/10.1007 /BF00964322 van der Kolk, B. A., Hodgdon, H., Gapen, M., Musicaro, R., Suvak, M. K., Hamlin, E., \& Spinazzola, J. (2016). A randomized controlled study of neurofeedback for chronic PTSD. PLoS ONE, 11(12), e0166752. http://dx.doi.org /10.1371/journal.pone.0166752

van der Kolk, B. A., McFarlane, A. C., \& Weisaeth, L. (1996). Traumatic stress: The effects of overwhelming experience on mind, body, and society (pp. 226-229). New York, NY: Guilford Press.

Vasterling, J. J., Brailey, K., Constans, J. I., \& Sutker, P. B. (1998). Attention and memory dysfunction in posttraumatic stress disorder. Neuropsychology, 12(1), 125-133. http://psycnet.apa.org/doi/10.1037/0894-4105.12.1.125

Vasterling, J. J., Duke, L. M., Brailey, K., Constans, J. I., Allain, A N., \& Sutker, P. B. (2002). Attention, learning, and memory performances and intellectual resources in Vietnam veterans: PTSD and no disorder comparisons. Neuropsychology, 16(1), 5-14. http://psycnet.apa.org/doi/10.1037/0894-4105.16.1.5

White, N. E., \& Richards, L. M. (2009). Alpha-theta neurotherapy and the neurobehavioral treatment of addictions, mood disorders and trauma. In T. H. Budzynski, H. K. Budzynski, J. R. Evans, \& A. Abarbanel (Eds.), Introduction to Quantitative EEG and Neurofeedback: Advanced Theory and Applications (2nd ed., pp. 143-166). San Diego, CA: Academic Press.

Wylie, M. S. (2004). The limits of talk. Psychotherapy Networker, 28(1), 30-36. http://dubielgray.com/wp-content/uploads/2014 108/Networker.pdf

Received: October 16, 2017

Accepted: November 2, 2017

Published: December 8, 2017 